\title{
Multi Tooth Uneven Cutting Behavior of High-speed Milling Cutter and Criterion for Milled Surface Topography
}

\author{
Jiang Bin*, Yao Guisheng, Zhang Shuai and Xu Tong
}

\begin{abstract}
National \& Local United Engineering Laboratory of High Efficiency Cutting and Tools, Harbin University of Science and Technology, Harbin, Heilongjiang, 150080, P.R. China
\end{abstract}

\begin{abstract}
With the cutting model of high-speed milling cutter in vibration condition, milling cutter vibration and effect of tooth error on cutting parameter are researched and high-speed milling cutter multi tooth uneven cutting behavior characterization methods are proposed. Use of high speed milling cutter cutting experiments and simulation results of surface topography, processing surface morphology characteristic parameters of cutter multi-tooth uneven cutting behavior response characteristics are revealed under the influence of milling cutter vibration and error, surface topography criterion is established in the cutting behavior of high speed milling cutter with multi-tooth, judgment method of multitooth uneven cutting behavior of high speed milling cutter with multi-tooth is proposed. Use the above criteria and evaluation method, reveal the formation mechanism of multi tooth uneven cutting, proposed compensation method of multi tooth uneven cutting behavior of high-speed milling cutter and do an experiment to verify the method.
\end{abstract}

Keywords: High-speed milling cutter, vibration, multi tooth cutting, uneven cutting behavior, milled surface topography.

\section{INTRODUCTION}

High-speed face milling cutter is a typical multi tooth high efficient cutting tool, widely used in finish machining of large structural component [1-3]. In the cutting process of high speed face milling cutter, under the influence of the centrifugal force vibration generated by quality eccentric of milling cutter, cutting force vibration generated by rotation cutting of multiple cutter tooth, machine tool vibration and the error between cutter tooth, cutting contact relationship of tool and workpiece is in unstable state [4-7], causing multiple cutter tooth cutting conduct inconsistency. Thus caused milling cutter multi-tooth uneven cutting problem is outstanding, it is easy to cause the processing surface quality of large parts deterioration.

When high-speed face milling cutter milling, on one hand, requirements with great metal removal rate to reduce the time of using machine, on the other hand, requirements with low cutting vibration and high quality of machining face [8-10]. Therefore, milling cutter commonly adopted the cutter tooth distribution with unequal blade spacing, and reduce the global error of milling cutter as possible, to improve machined surface quality of high speed milling cutter. The above methods can efficient inhibition the cutting force vibration by disperse the energy of cutting vibration. By improve the installation precision of cutter tooth, decreased the centrifugal force vibration caused by mass eccentricity. To a certain extent solved the problem of milling cutter multi tooth uneven cutting and machined surface quality decline
Studies have shown that, the high-speed milling cutter vibration that influenced milling cutter multi-tooth uneven cutting. Not only including the vibration behavior decided by dominant frequency, but also include other vibration signals, Such as machine tool vibration and the cutting chatter. This part vibration also influence cutter cutting behavior [11-14]. At the same time, using the method of improving the install precision of milling cutter tooth, control the global error of milling cutter and reducing multi tooth uneven cutting. Not only make milling cutter manufacturing and debugging difficulty double increased, and the degree of solving problems is limited.

High-speed milling cutter multi-tooth uneven cutting is the results of vibration milling cutter and cutter tooth error comprehensive effect. There are several design method to cutter tooth unequal spacing distribution, not every design method can solve the problem of multi tooth uneven cutting effectively. Randomness and diversity of cutter tooth distribution in design, make it lack of an adequate basis in the control of cutter tooth cutting behavior.

The existing methods mainly adopts single factor control variable method, aiming at the high-speed milling cutter's cutting behavior characteristics which influence milled surface topography obviously to do a study and modeling, less comprehensive considering the influence of the interaction among multi-characteristic vector on the cutting behavior of cutter's each tooth. And unable to control the consistency of cutting behavior among cutter teeth effectively.

At this background, this paper aiming at the above problems that exist in high-speed milling cutter multi-tooth cutting, through characterization of high-speed milling cutter multi tooth uneven cutting behavior, exploring the influence mechanism of high-speed milling cutter's vibration, knife 
tooth's error on knife tooth's cutting behavior, revealing the relationship between multi tooth cutting behavior and milled surface topography, putting forward the method to control high-speed milling cutter multi tooth uneven cutting behavior.

\section{MULTI TOOTH UNEVEN CUTTING BEHAVIOR CHARACTERIZATION OF HIGH-SPEED MILLING CUTTER}

According to the theory of vibration system and existing milling model, models high-speed milling cutter under the condition of vibration as Figs. $(\mathbf{1}, \mathbf{2})$ shows. In the figure, coordinate system origin is specified point on the machine, $x$ is in the direction of row spacing, $y$ is in the cutter feeding direction, $z$ is in the axial direction, $A^{x}{ }_{C i}(t)$ and $A^{y}{ }_{C i}(t)$ is vibration amplitude along the $x$ and $y$ direction, $\Delta_{i, i+1}^{d}$ is radial error between any two adjacent teeth $(\mathrm{mm})$, and $\Delta^{L}{ }_{i, i+1}$ is axial error of the arbitrary two adjacent teeth ( $\mathrm{mm})$, two adjacent tooth $i$ and $i+1$ error before $i$ is measurement benchmark.

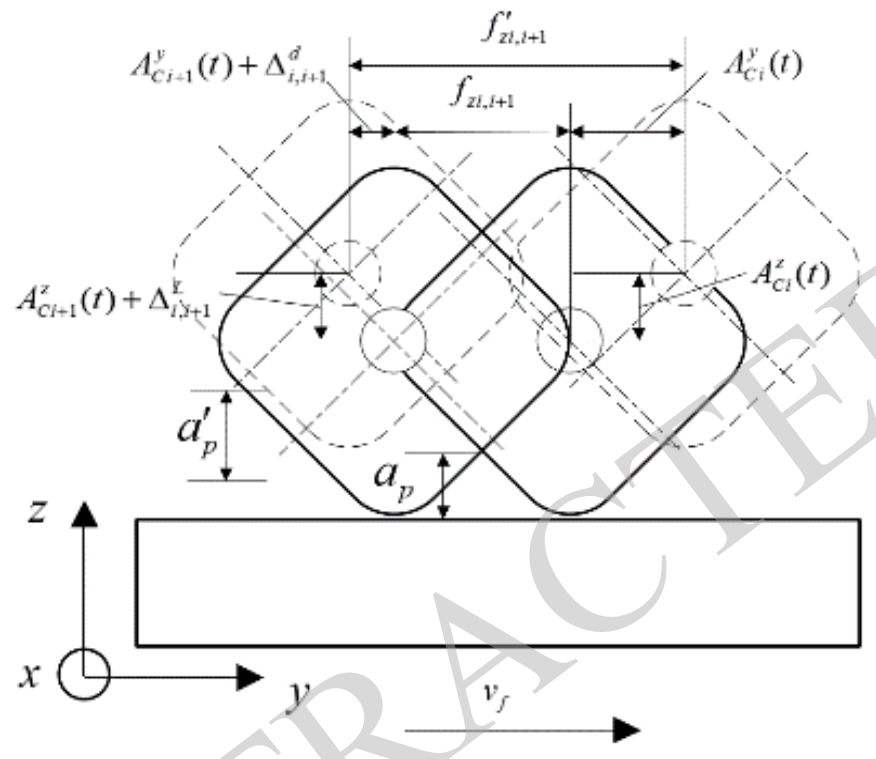

Fig. (1). Cutter behavior on xoz plane.

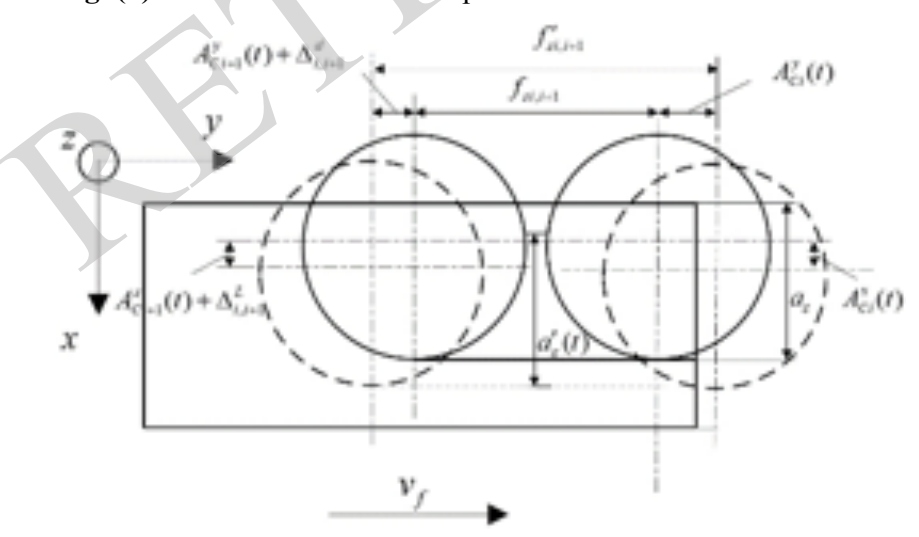

Fig. (2). Cutter behavior on xoy plane.

High-speed milling cutting parameters change with time on the condition of vibration from Fig. (1) are as follows.

$f_{z i, i+1}^{\prime}=\frac{v_{f} \theta_{i, i+1}}{2 \pi n}+A_{C i}^{y}(t)+A_{C i+1}^{y}(t)+\Delta_{i, i+1}^{L}$
$a_{p}^{\prime}(t)=a_{p}+A_{C i}^{y}(t)+A_{C i+1}^{y}(t)+\Delta_{i, i+1}^{L}$

$a_{\varepsilon}^{\prime}(t)=a_{\varepsilon}+A_{C i}^{x}(t)+A_{C i+1}^{x}(t)+\Delta_{i, i+1}^{d}$

When high speed cut, under the influence of milling vibration and tooth error, cutter blade cutting parameters change constantly, and lead to generate multi tooth uneven cutting behavior.

Use the model to identify the multi tooth uneven cutting behavior of high-speed milling cutter as Fig. (3) shows. In the Fig. (3), $f_{x}, f_{y}$ and $f_{z}$ are vibration main frequency respectively along the $x, y$ and $\mathrm{z}$ direction, and $A_{x}, A_{y}$ and $A_{z}$ are vibration amplitude respectively along the $x, y$ and $\mathrm{z}$ direction.

The method can identify the multi tooth uneven cutting behavior of high-speed milling cutter along different direction through cutter tooth error distribution characteristics, using time and frequency domain test results along the row spacing, feed direction and axial vibration in the process of high speed cutting, and according to the effect of cutter and blade vibration error on tooth cutting.

\section{CRITERION FOR MILLED SURFACE TOPO- GRAPHY OF HIGH-SPEED MILLING CUTTER MULTI TOOTH UNEVEN CUTTING BEHAVIOR}

Use high-speed milling cutter with diameter of $63 \mathrm{~mm}$ and four teeth distribution with the same pitch. Cutting 45 steel under the condition of $1011 \mathrm{rpm}$ speed, $0.08 \mathrm{~mm} / \mathrm{z}$ per tooth, $0.3 \mathrm{~mm}$ cutting depth and $40 \mathrm{~mm}$ cutting width. Obtaining the vibration characteristics of cutter and milled surface topography as Table 1 and Fig. (4) shows.

Using the vibration test results of cutting experiment, considering the influence of milling cutter vibration and cutter tooth error. Use the type (1) (3) to solve cutter tooth cutting behavior, simulation with high-speed milling milled surface topography. Revealed the relationship between cutting behavior of cutter tooth and milled surface morphology under the influence of milling cutter vibration and cutter tooth error as Fig. (5) shows.

Simulation of high-speed milling cutter machining surface topography and experimental comparative analysis results show that cutter's multi tooth uneven cutting caused by the inconformity of vibration frequency and cutting frequency of knife tooth makes milled surface topography and its distribution change obviously.

According to the above results, use high-speed milling cutter vibration, error and cutting behavior parameters of tooth, produce influence characteristic to milled surface residual height $R_{\max , i+1}$, entering angle $\kappa_{r}$, and spacing of adjacent residual height $\Delta L_{i+1}$. Establish the judgment criteria of milled surface morphology of high-speed milling cutter multi tooth cutting behavior.

$$
\begin{aligned}
& R_{\max i+1}=\frac{f_{z i, i+1}^{\prime}+A_{C i}^{z}(t) \cot k_{r}+\left(\Delta_{i, i+1}^{L}+A_{C i+1}^{z}(t)\right) \cot k_{r}^{\prime s}}{\cot k_{r}+\cot k_{r}^{\prime s}} \\
& k_{r}^{\prime s}=k_{r}^{\prime} \mp \Delta k_{r}=k_{r}^{\prime} \mp 2 \operatorname{atctan} \frac{A_{C i+1}^{z}(t)-A_{C i}^{z}(t)}{A_{C i+1}^{y}(t)-A_{C i}^{y}(t)}
\end{aligned}
$$


$\Delta L_{i+1}=f_{z i, i+1}^{\prime}=\frac{v_{f} \theta_{i, i+1}}{2 \pi n}+A_{C i}^{y}(t)+A_{C i+1}^{y}(t)+\Delta_{i, i+1}^{d}$
Use the above judgment criteria for high speed milling cutter multi tooth uneven cutting behavior evaluation as Fig. (6) shows.

Since cutter error caused characteristics of- periodic of
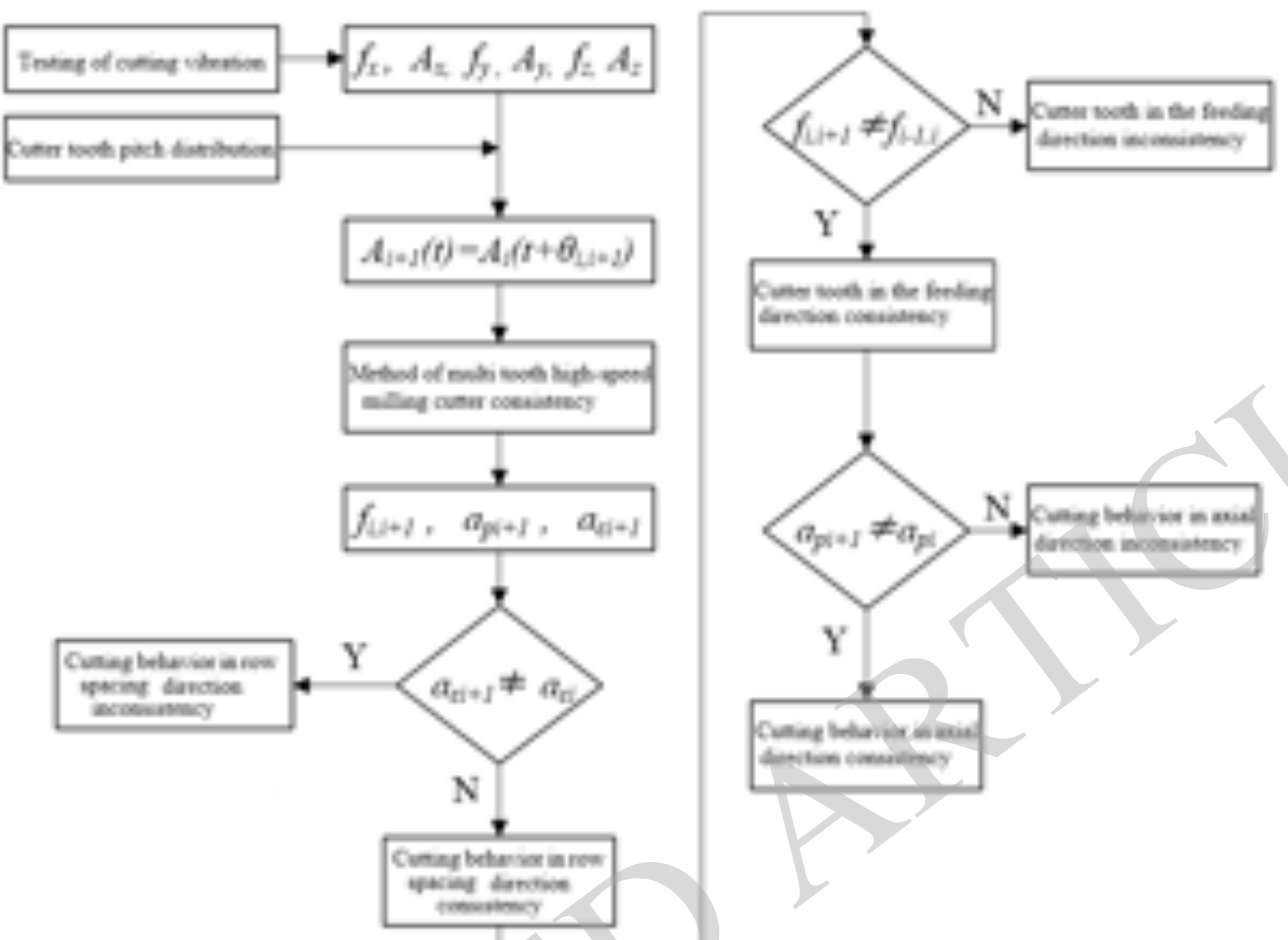

Fig. (3). Recognition method of milling cutter consistency.

Table 1. Characteristic of cutter vibration.

(1-1)

\begin{tabular}{|c|c|c|c|}
\hline \multicolumn{2}{|c|}{ Time Domain Feature of Cutter Vibration } & \multicolumn{2}{c|}{ Characteristics in Frequency Domain of Cutter Vibration } \\
\hline \hline Amplitude in row spacing direction, $\Delta A_{x}\left(\mathrm{~m} / \mathrm{s}^{2}\right)$ & 12.31 & Number of dominant frequency in row spacing direction, $m_{x}$ & 4 \\
\hline Waveform factor in row spacing direction, $K_{f x}$ & 1.29 & First dominant frequency in row spacing, $f_{x}(\mathrm{~Hz})$ & 5800.78 \\
\hline Waveform kurtosis in row spacing direction, $K_{x}$ & 3.14 & Amplitude in row spacing direction, $A_{x}\left(\mathrm{~m} / \mathrm{s}^{2}\right)$ & 0.64 \\
\hline
\end{tabular}

(1-2)

\begin{tabular}{|c|c|c|c|}
\hline \multicolumn{2}{|c|}{ Time Domain Feature of Cutter Vibration } & \multicolumn{2}{|c|}{ Characteristics in Frequency Domain of Cutter Vibration } \\
\hline Amplitude in axial direction, $\Delta A_{z}\left(\mathrm{~m} / \mathrm{s}^{2}\right)$ & 13.48 & Number of dominant frequency in axial direction, $m_{z}$ & 4 \\
\hline Waveform factor in axial direction, $K_{f z}$ & 1.25 & First dominant frequency in axial direction, $f_{z}(\mathrm{~Hz})$ & 7675.78 \\
\hline Waveform kurtosis in axial direction, $K_{z}$ & 2.86 & Amplitude in axial direction, $A_{z}\left(\mathrm{~m} / \mathrm{s}^{2}\right)$ & 0.33 \\
\hline
\end{tabular}

\section{(1-3)}

\begin{tabular}{|c|c|c|c|}
\hline \multicolumn{2}{|c|}{ Time Domain Feature of Mill Vibration } & \multicolumn{2}{c|}{ Characteristics in Frequency Domain } \\
\hline \hline Amplitude in feed direction, $\Delta A_{y}\left(\mathrm{~m} / \mathrm{s}^{2}\right)$ & 12.07 & Number of dominant frequency in feed direction, $m_{y}$ & 3 \\
\hline Waveform factor in feed direction, $K_{f y}$ & 1.26 & First dominant frequency in feed direction, $f_{y}(\mathrm{~Hz})$ & 5996.09 \\
\hline Waveform kurtosis in feed direction, $K_{y}$ & 2.86 & Amplitude in feed direction, $A_{y}\left(\mathrm{~m} / \mathrm{s}^{2}\right)$ & 0.47 \\
\hline
\end{tabular}


tooth uneven cutting behavior, that will not change processing surface texture. Changes in the direction of milled surface texture is mainly because uneven cutting caused by milling cutter vibration. Therefore, this method first using the characteristic to judge if there is vibration influence, then respectively using the criterion of milled surface residual height and distance to judge uneven cutting characteristic of cutter tooth.

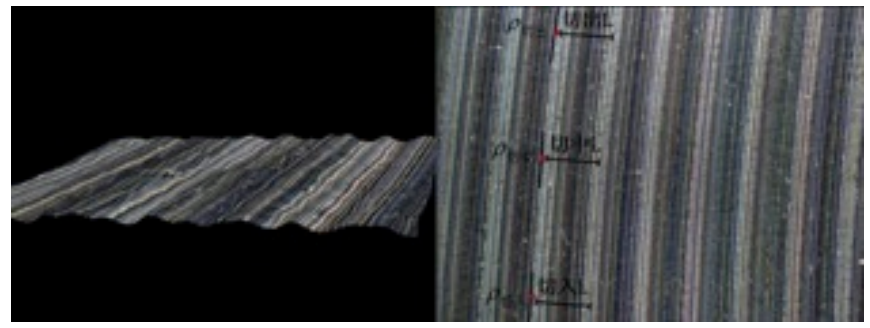

Fig. (4). High speed milling surface topography.

\section{COMPENSATION METHOD ON MULTI TOOTH UNEVEN CUTTING BEHAVIOR OF HIGH-SPEED MILLING CUTTER}

According to type (1) (3), the misalignment between vibration frequency of cutter and cutting frequency of tooth causes the change of cutting parameter of adjacent tooth. It is one of the important reasons of tooth uneven cutting. When vibration frequency of cutter and cutting frequency satisfy is integer times, the vibration waveform of each tooth in the same position are basically identical, and uneven cutting behavior of tooth caused cutter vibration reducing greatly. Hereby, putting forward compensation method on multi tooth uneven cutting behavior of high-speed milling cutter.

$\theta_{i, i+1}=\frac{N_{1} n \pi}{30 f_{x}}=\frac{N_{2} n \pi}{30 f_{y}}=\frac{N_{3} n \pi}{30 f_{z}}$

In the type, $\theta_{i, i+1}$ is adjacent tooth $i$ and $i+1, N_{l}, N_{2}, N_{3}$ are integers, $n$ is speed, $f_{x}, f_{y}, f_{z}$ are main vibration frequency of cutter along row spacing direction, cutter feeding direction, and axial direction.

Vibration of high-speed milling cutter includes not only the vibration behavior determined by the main frequency of vibration, but also other vibration signals, such as vibration of machine and cutting flutter also influence cutting behavior of cutter. Therefore, pitch compensation method can only exist integer-times relationship between tooth cutting frequency and one of vibration signal in a variety of vibration signals, but unable to compensate the uneven cutting caused by cutter

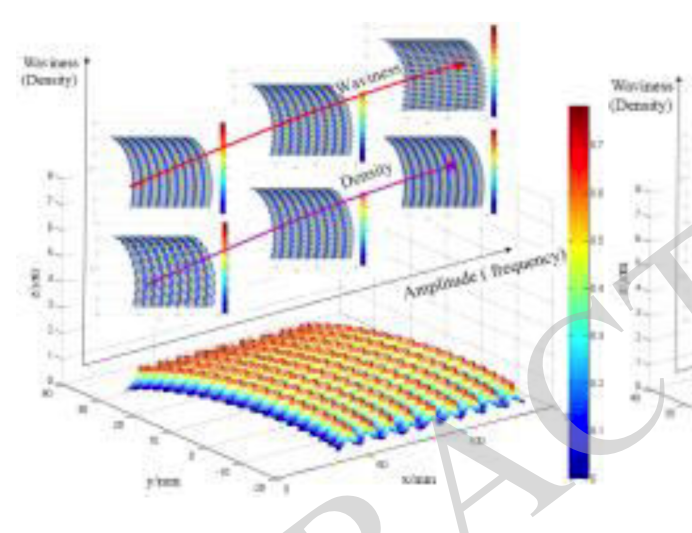

(a) Feed direction

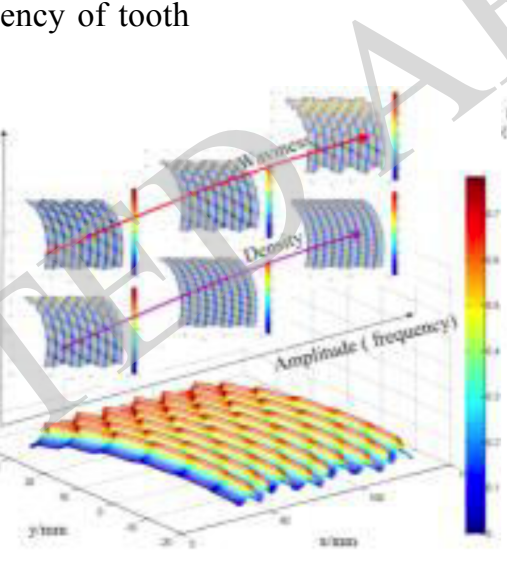

(b) Row spacing

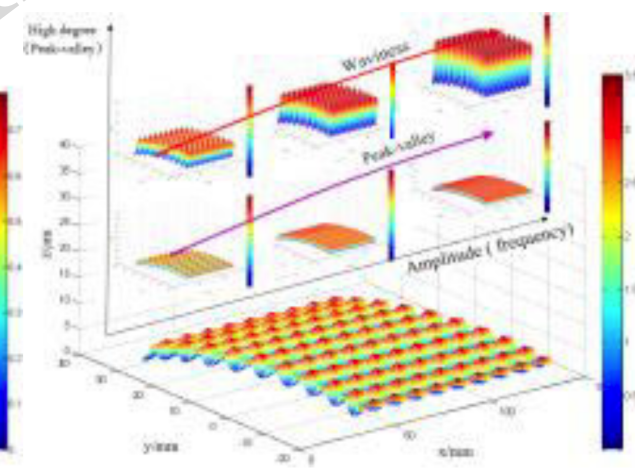

(c) Axial direction

Fig. (5). Simulation results of milled surface topography.

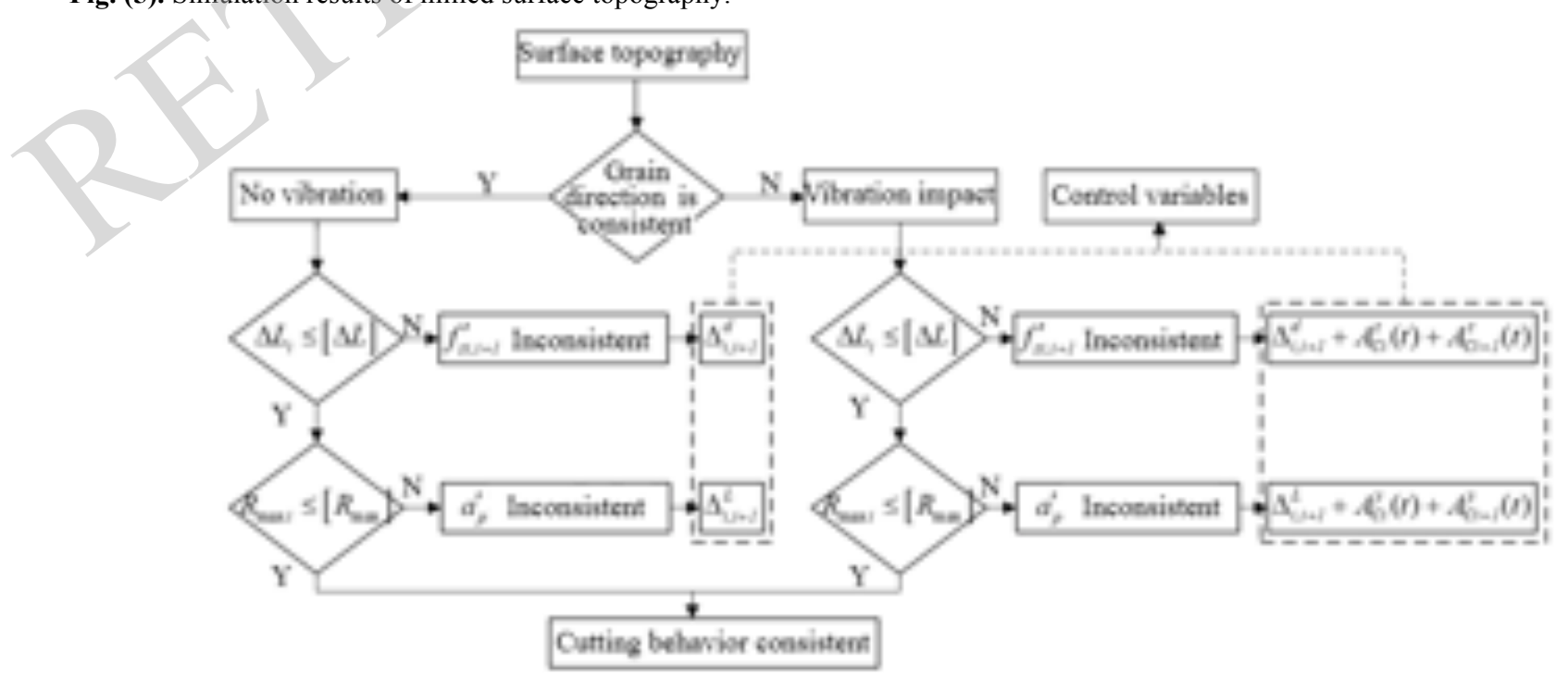

Fig. (6). Evaluation method of multi-tooth cutting behavior. 


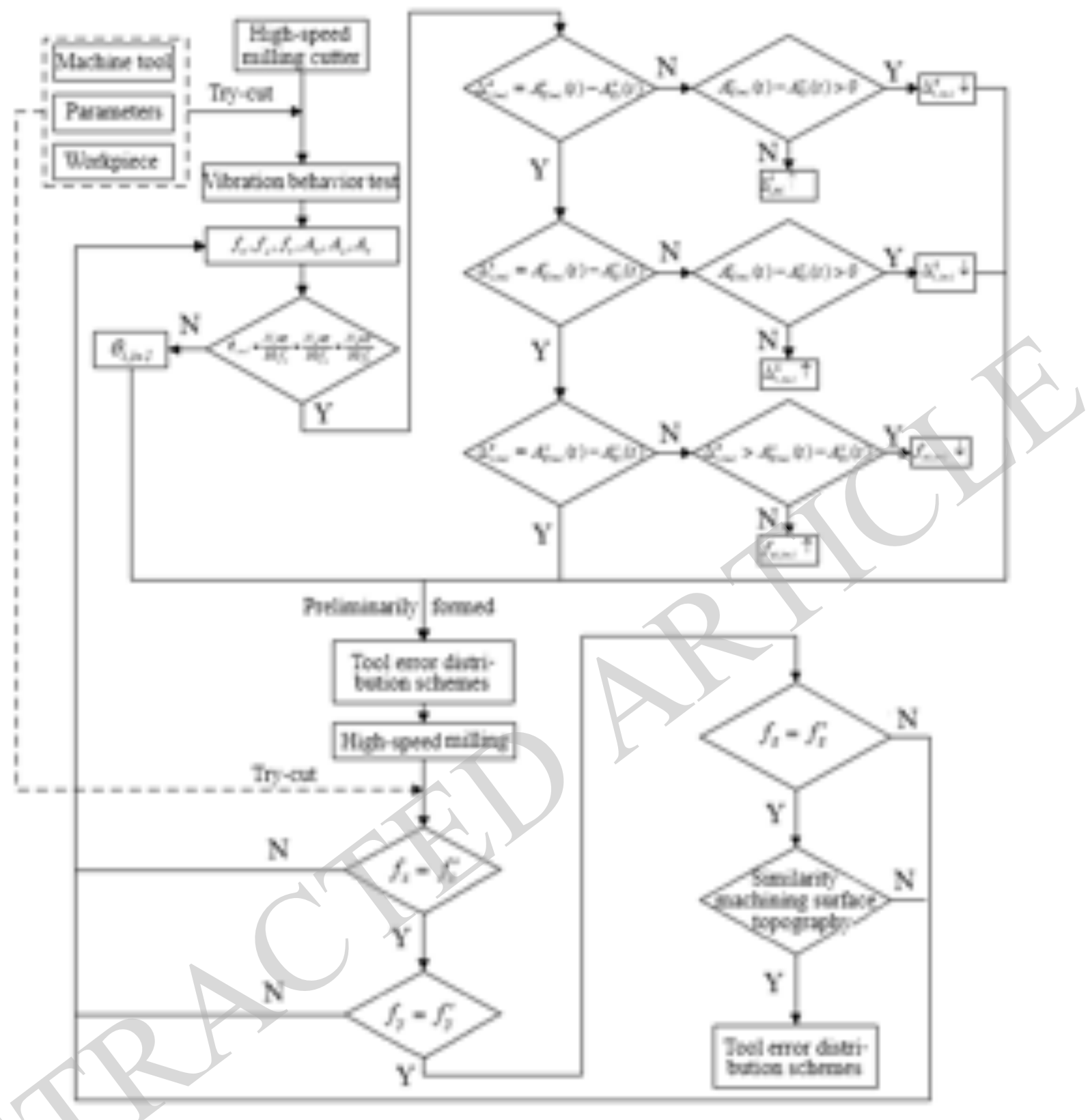

Fig. (7). Compensation method of uneven cutting.

vibration of multiple main frequency.

To this end, on the basis of milling cutter tooth pitch compensation, according to the pitch and speed adjusted milling vibration test results, for high speed milling cutter tooth more uneven cutting behavior of secondary identification, by type (1) (3) satisfying cutter tooth cutting behavior is more relevant and distribution conditions of cutter tooth error, the use of processing and surface morphology criterion for blade design error and its distribution, two actions of the cutter tooth more uneven cutting times compensation as Fig. (7) shows.

\section{THE EXPERIMENT OF MULTI TOOTH CUTTING BEHAVIOR OF HIGH-SPEED MILLING CUTTER}

In the speed is $1011 \mathrm{rpm}$, feed per tooth is $0.08 \mathrm{~mm} / \mathrm{z}$, cutting depth is $0.3 \mathrm{~mm}$, cutting width is $40 \mathrm{~mm}$, doing the experiment of high-speed face milling cutter whose diameter is $63 \mathrm{~mm}$ cutting steel 45 . Based on the vibration and milled surface topography's test results of high-speed face milling cutter whose diameter is $63 \mathrm{~mm}$ and four teeth uniform distribution cutting steel 45 , using the compensation method shown in Fig. (7), designing the cutter's pitch and knife tooth's error, the results are shown in Table $\mathbf{2}$, and cutter A is high-speed face milling cutter whose diameter is $63 \mathrm{~mm}$ and four teeth uniform distribution, cutter B is the cutter after error correction.

Under the condition of the same cutting parameters, using cutter A and B in Table 2 cutting steel 45, obtaining the experimental results of cutter's vibration characteristics and milled surface topography's characteristics are Figs. (810) shows. 
Table 2. The structure parameters of milling cutter.

\begin{tabular}{|c|c|c|c|}
\hline \multicolumn{2}{|c}{ Cutter } & A & B \\
\hline \hline \multirow{4}{*}{ Pitch ( $)$} & $\theta_{12}$ & 90 & 82.41 \\
\cline { 2 - 4 } & $\theta_{23}$ & 90 & 87.47 \\
\cline { 2 - 4 } & $\theta_{34}$ & 90 & 92.53 \\
\cline { 2 - 4 } & $\theta_{41}$ & 90 & 97.59 \\
\hline \multirow{3}{*}{ Axial error (mm) } & $L_{12}$ & 0.019 & -0.015 \\
\cline { 2 - 4 } & $L_{23}$ & 0.012 & 0.015 \\
\cline { 2 - 4 } & $L_{34}$ & -0.002 & -0.015 \\
\cline { 2 - 4 } & $L_{41}$ & -0.029 & 0.015 \\
\hline \multirow{3}{*}{ Radial error (mm) } & $d_{12}$ & 0.11 & 0.014 \\
\cline { 2 - 4 } & $d_{23}$ & 0 & -0.014 \\
\cline { 2 - 5 } & $d_{34}$ & 0 & -0.014 \\
\cline { 2 - 5 } & $d_{41}$ & -0.11 & 0.014 \\
\hline
\end{tabular}

This experimental results show that the change of cutter's pitch causes cutting vibration's frequency domain wave changing, but does not cause the main properties of the cutter's vibration changing. After adopting the compensation method shown in Fig. (7), the scallop height of cutter's milled surface topography decreases from $12.76 \mu \mathrm{m}$ to 0.461 $\mu \mathrm{m}$, the unevenness of adjacent scallop height spacing falls from $7.75 \% \sim 0.5 \%$. The compensation effect of cutter's multi tooth uneven cutting behavior is remarkable. The consistency of high-speed milling cutter's multi tooth uneven cutting behavior and machined surface quality of high-speed milling are improved obviously.

\section{CONCLUSION}

Building the feature model of knife tooth's cutting behavior under the condition of vibration, revealing the influence of cutter's vibration and error on knife tooth's cutting parameter, realizing the quantitative description of cutter's multi-tooth cutting behavior, proposing the recognition method of high-speed milling cutter's multitooth uneven cutting behavior.

The contrast analysis results of high-speed milling cutter's cutting experiment and milled surface topography's show that cutter's multi-tooth uneven cutting caused by the inconformity of cutter's vibration frequency and knife tooth's cutting frequency makes milled surface topography's and its distribution change obviously. The processing surface residual height and its spacing caused by two adjacent knife tooth have significant response to cutter's multi-tooth uneven cutting. On this basis, building the milled surface topography's criterion of high-speed milling cutter's multitooth cutting behavior.

The evaluation results of multi tooth uneven cutting behavior of high-speed milling cutter show that speed and blade pitch distribution not only affects cutter vibration, but

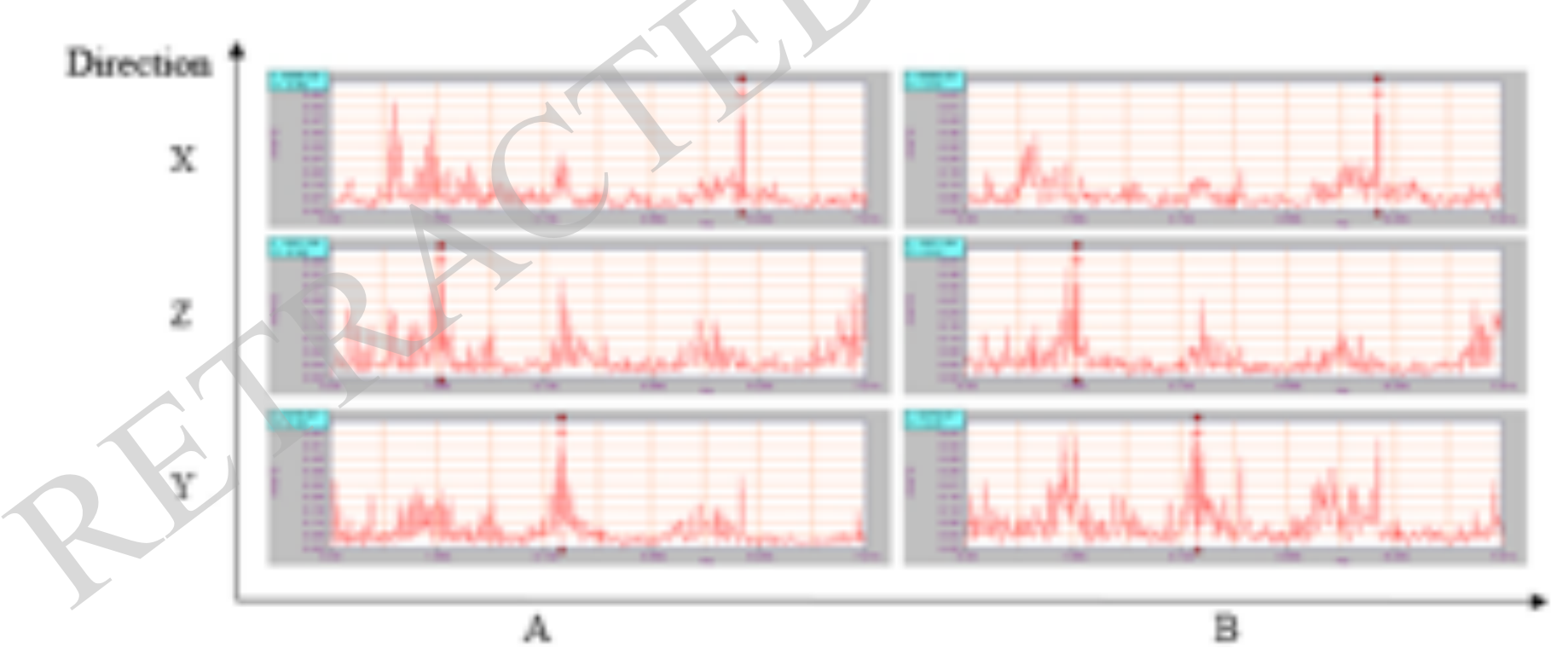

Fig. (8). Frequency domain characteristics of vibration behavior.

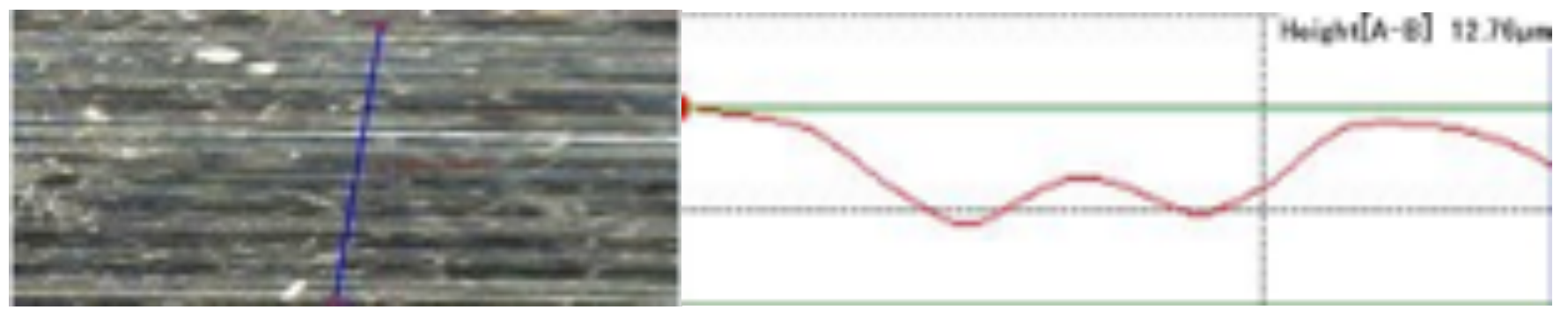

Fig. (9). Milled surface topography of cutter A. 


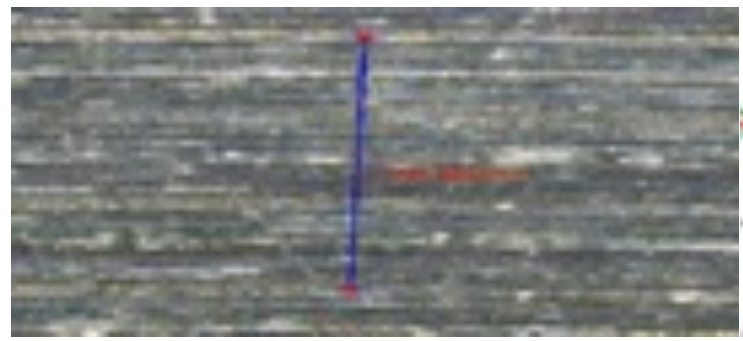

Fig. (10). Milled surface topography of cutter B.

also directly determine the blade cutting frequency. Vibration frequency of milling cutter and the tooth cutting frequency is integer times inconsistent or not, and multiple frequency vibration milling cutter is the basic reason of multi tooth uneven cutting of high-speed milling cutter.

The compensation method of multi tooth uneven cutting behavior of high-speed milling cutter is put forward, according to the deficiency of this method, propose multi tooth uneven cutting behavior of secondary compensation method.

The experiment results show that high-speed milling cutter improve the surface morphology and distribution uniformity and multi tooth uneven cutting behavior of highspeed milling cutter had a remarkable effect on the basis of cutter tooth pitch compensation, through the cutter tooth error and its distribution design for secondary compensation.

\section{CONFLICT OF INTEREST}

The authors confirm that this article content has no conflict of interest.

\section{ACKNOWLEDGEMENTS}

This work was financially supported by the National Natural Science Foundation of China (51375124).

\section{REFERENCES}

[1] Z.Q. Li, Q. Liu, and S.M. Yuan, "Prediction of dynamic cutting force and regenerative chatter stability in inserted cutters milling," Chinese Journal of Mechanical Engineering, vol. 3, pp. 555-563, 2013.

[2] B. Jiang, Method Research on High Speed Milling Cutter Stability and Structure Optimization Design, Harbin University of Science and Technology, 2008.
[3] X. Q. Yuan, X.J. Jun and S.X. Zhang, "MR-TMD Damping vibration control system of continuous box girder bridge," Vibration and Shock, vol. 20, pp. 153-157, 2012.

[4] Q. Guo, Y.W. Sun and D.M. Guo, "New mathematical method for the determination of cutter runout parameters in flat-end milling," Chinese Journal of Mechanical Engineering, vol. 5, pp. 947-952, 2013.

[5] H. Li, Y.P. Zhang and F.L. Liu, "Research on effect of unequal blade spacing milling cutter to end milling vibration," Mechanical Science and Technology, vol. 3, pp. 408-417, 2012.

[6] Y. X. Qin, Z. Q. Liu and A. J. Tang, "Research summary of high speed cutting stability," Tool Engineering, vol. 9, pp. 3-8, 2011.

[7] J. Zhou, Research on Aluminum 7050-T7451 Cutting Processing Mechanism and Surface Integrity, Shandong University, 2011.

[8] C. G. Fan, The Surface Morphology Research on High-Speed Milling of Aluminum Alloy 7475, Harbin University of Science and Technology, 2011.

[9] P. Vanherek, "Increasing milling machine productivity by use of cutter with non-constant cutting edge pitch," In: Proceedings of Advanced MTDR Conference, 2011, pp. 947-960.

[10] H. Ding, Investigtion on Cutting Mechanisms and Experiments in Two-dimensional Vibration-assisted Micro Milling, Harbin Institute of Technology, 2012.

[11] K.P. Wang, W.Y. Tang and H.X. Xue, "Time domain analysis approach for riser vortex-induced vibration based on forced vibration test data," In: Proceedings of the International Conference of Offshore Mechanics \& Arctic Engineering OMAE, vol. 7, 2013.

[12] H. Jiang, X.H. Hua, and G. Meng, "Active vibration control of milling processes," In: Proceedings of the ASME International Mechanical Engineering Congress and Exposition, vol. 8, pp. 947955, 2010.

[13] W.Y. Bao, and I. N. Tandsel, "Modeling micro-end-milling operations. Part I:Analytical cutting force model," International Journal of Machine Tools and Manufacture, vol. 40, pp. 21552173, 2000.

[14] C.F. Li, X.M. Lai and H.T. Li, "Modeling of three-dimensional cutting forces in micro-end- milling," Journal of Microbiology, vol. 17, pp. 671-678, 2007. 